\title{
ELECTRICAL AND OPTICAL TRANSDUCTION OF SINGLE-CRYSTAL 3C-SiC COMB-DRIVE RESONATORS IN A SiC-ON-INSULATOR (SiCOI) TECHNOLOGY \\ J. P. McCandless ${ }^{1,2}$, J. Lee ${ }^{l}$, H.-I. Kuo ${ }^{1,3}$, V. Pashaei ${ }^{1}$, M. Mehregany ${ }^{1}$, C. A. Zorman ${ }^{1}$, and P. X.-L. Feng ${ }^{1 *}$ ${ }^{1}$ Electrical Engineering, Case Western Reserve University, Cleveland, OH 44106, USA
}

\begin{abstract}
We report on a study of electrical and optical transduction of single-crystal $3 \mathrm{C}$-SiC comb-drive lateral resonators. These $\mathrm{SiC}$ resonators were surface micromachined from high-quality singlecrystal 3C-SiC films on SiC-on-insulator (SiCOI) substrates made using a bonding-free transfer technique previously reported by our team. We have measured multiple modes in such resonators using all-electrical transduction with capacitive actuation and detection, as well as optical readout. We have observed the onset of nonlinearity for optical displacement transduction in the 'knifeedge' measurement scheme, which helps to precisely calibrate the vibrational amplitude of each mode. These devices, along with such signal transduction schemes, shall enable new multimode and integrated resonant sensors from single crystalline $3 \mathrm{C}-\mathrm{SiC}$.
\end{abstract}

\section{INTRODUCTION}

Silicon carbide $(\mathrm{SiC})$ is a technologically important material owing to its exceptional electrical, optical, thermal, and mechanical properties. $\mathrm{SiC}$ is a wide bandgap polymorphic semiconductor $\left(E_{\mathrm{g}} \approx 2.36-3.23 \mathrm{eV}\right.$, depending on the crystalline polytype) especially useful for power electronics and hightemperature devices. It is also a natural near-zero-epsilon material at $\sim 10.3 \mu \mathrm{m}$ [1], which offers promises for useful optical and photonic devices. Besides being chemically inert, radiation hard, and high temperature capable, it lends itself to bulk and surface micro/nanomachining [2,3], allowing for the fabrication of robust and versatile $\mathrm{SiC}$ micro/nanoelectromechanical systems (MEMS/ NEMS). Furthermore, its excellent mechanical properties (e.g., Young's modulus, $\left.E_{\mathrm{Y}} \sim 450 \mathrm{GPa}\right)$ make it attractive for enabling resonant MEMS/NENS operating at radio frequencies (RF). While over two hundred different polytypes of $\mathrm{SiC}$ have been identified, only $4 \mathrm{H}-, 6 \mathrm{H}-$, and $3 \mathrm{C}-\mathrm{SiC}$ are available in single crystalline form and have established manufacturing protocols for large wafers.

It is much desirable to develop single-crystal $\mathrm{SiC}$-on-insulator (SiCOI) technologies for emerging applications in electronics, transducers, and photonics. However, such developments have been particularly challenging because the most widely used $\mathrm{SiC}$ single crystals, i.e., $4 \mathrm{H}-\mathrm{SiC}$ and $6 \mathrm{H}-\mathrm{SiC}$, come in bulk substrates instead of thin films; and their growth or deposition on insulators (such as $\mathrm{SiO}_{2}$ ) has been elusive. To date, only polycrystalline 3CSiCOI wafers are available with high-yield growth [2,3]. Mainstream techniques for realizing single-crystal SiC-oninsulator, such as wafer bonding or 'Smart-Cut', suffer from lowyield bonding and transfer of the single-crystal $\mathrm{SiC}$ thin film to the insulating layer, and high-cost ion implantation processes $[4,5]$.

We have regularly reported progresses in surface micro/ nanomachined polycrystalline $\mathrm{SiC}$ MEMS/NEMS devices since the early 2000's. In comparison, our studies on surface micro/ nanomachined single-crystal $\mathrm{SiC}$ devices have been far fewer. In 2003, we described a method to produce single crystalline $3 \mathrm{C}-\mathrm{SiC}$ on insulator substrates using a transfer technique that involves growing a polycrystalline silicon (poly-Si) layer to a wafer-scale thickness by CVD on top of an oxide capped 3C-SiC film followed by etch back of the single crystal handle wafer used for $3 \mathrm{C}-\mathrm{SiC}$ epitaxy [6]. This bonding-free method was used to produce 100 mm-diameter 3C-SiCOI substrates with $100 \%$ transfer yield. To demonstrate their utility in surface micromachining, comb-drive lateral resonators were fabricated from these substrates and characterized for resonant behavior. In this work we revisit the comb-drive lateral resonators fabricated from single crystalline $3 \mathrm{C}$ SiCOI. Specifically, we study their resonance characteristics using both electrical and optical transduction techniques.

\section{RESULTS AND DISCUSSIONS}

The devices in this work are folded-beam lateral comb-drive MEMS resonators. The thicknesses of the $3 \mathrm{C}-\mathrm{SiC}$ and thermallygrown $\mathrm{SiO}_{2}$ layers are $1.5 \mu \mathrm{m}$ and $1.7 \mu \mathrm{m}$, respectively. Figure 1 briefly illustrates the fabrication process. An Al etch mask is sputtered onto the surface of the $3 \mathrm{C}$-SiCOI wafer and patterned using standard lithographic techniques. Subsequently, the $3 \mathrm{C}-\mathrm{SiC}$ layer is etched using an RIE process at 3 Torr and $150 \mathrm{~W}$. The chemistry recipe used includes $\mathrm{O}_{2}, \mathrm{CHF}_{3}$, and He with flow rates of $22.5,5.6$, and $55 \mathrm{sccm}$, respectively. The etch process is anisotropic, but not selective to the Si-containing layers, thus etching not only the $\mathrm{SiC}$ layer, but also the $\mathrm{SiO}_{2}$ layer and $\sim 5 \mu \mathrm{m}$ of the polysilicon substrate. After etching, the Al etch mask is removed with an $\mathrm{Al}$ wet etchant, and the devices are released with a $5 \mathrm{~min} \mathrm{HF}$ etch of the buried oxide layer. Figure 2 shows the images and SEM images of representative device designs.
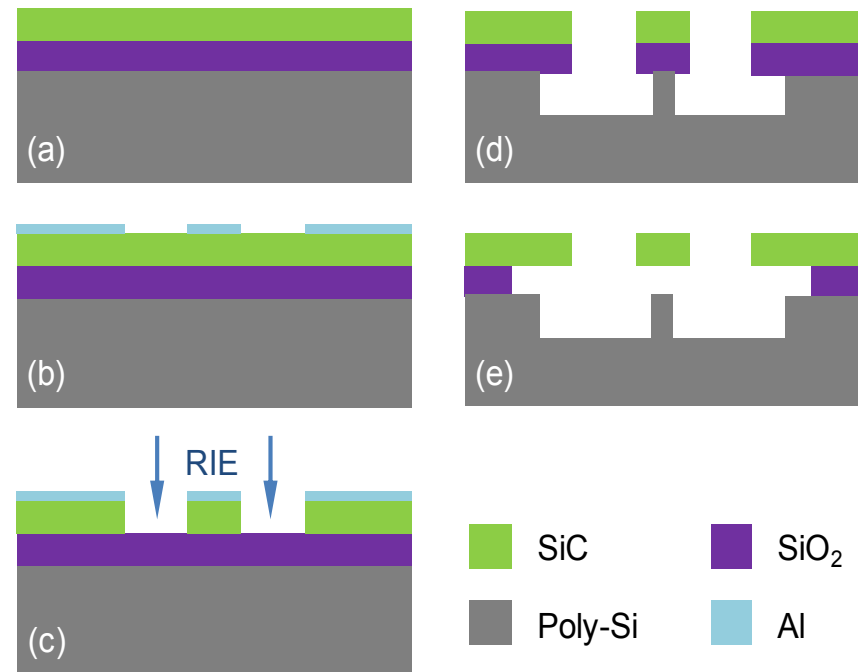

Figure 1: The 3C-SiCOI comb-drive resonator fabrication process. (a) The single-crystal 3C-SiCOI sample. (b) The Al etch mask is patterned by standard lithographic techniques. (c) SiC is etched using RIE. (d) Removal of Al etch mask. (e) The comb-drive resonator is released via a timed wet etch of the buried oxide layer.

Resonance behaviors of the $3 \mathrm{C}-\mathrm{SiC}$ comb-drive resonators are characterized using a motion detection apparatus which enables the measurement of device resonances with both optical and electrical transduction schemes. That is, it allows for measurements that involve (i) electrical actuation with electrical detection, and (ii) electrical actuation with optical detection of multimode in-plane and out-of-plane responses, simultaneously, via interferometric readout and 'knife-edge' techniques [7]. All measurements are performed in moderate vacuum ( 20mTorr) and at $\sim 300 \mathrm{~K}$. 

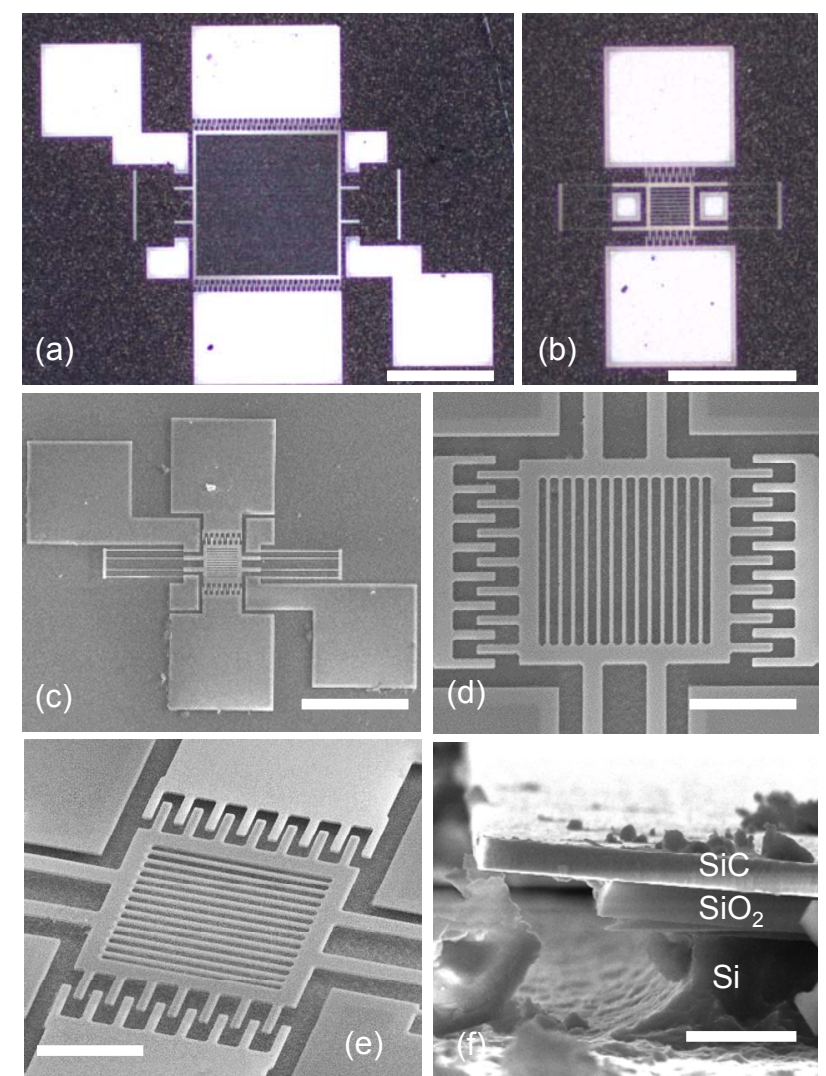

Figure 2: Fabricated comb-drive resonators based on 3C-SiCOI. (a)-(b) Optical images. (c)-(e) SEM images. (f) Side view SEM image of fabricated 3C-SiCOI electrical bonding pads, clearly showing the $\mathrm{SiC}, \mathrm{SiO}_{2}$, and Si layers. Scale bars: $300 \mu \mathrm{m}$ in panels (a)-(c), $40 \mu \mathrm{m}$ in panels (d)-(e), and $5 \mu \mathrm{m}$ in panel $(f)$.

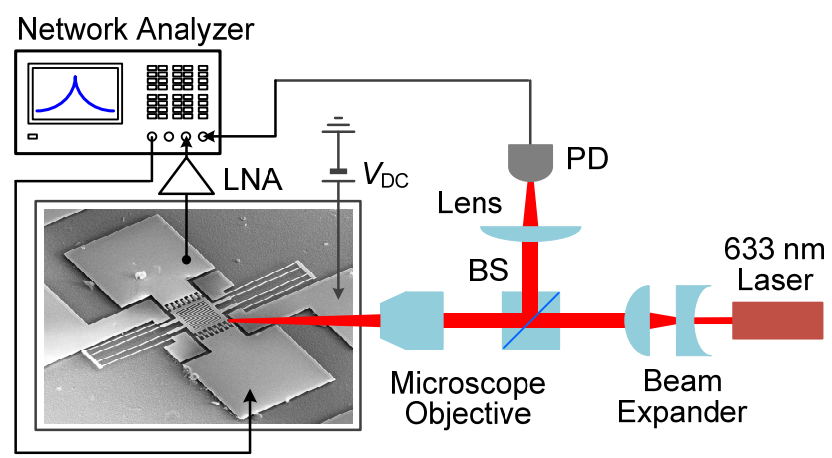

Figure 3: Simplified illustration of the combined optical and electrical resonance detection system. A network analyzer is used to capacitively drive the resonator. Resonances are detected using both optical and electrical transductions. The shuttle is polarized by a DC power supply. A low-noise voltage preamplifier with $40 \mathrm{~dB}$ gain is employed in the electrical detection scheme.

In the optical motion transduction scheme, we employ a customized home-built detection system (see Fig. 3). It can detect both out-of-plane and in-plane resonant motions through optical interferometry and the 'knife-edge' effects, respectively.

To attain the optical detection, a $633 \mathrm{~nm}$ laser is focused on devices using a $50 \times$ microscope objective. The typical on-device spot size in the system is $\sim 1 \mu \mathrm{m}$. To efficiently transduce in-plane motion using the 'knife-edge' technique, the laser spot is placed on the boundary of the comb-drive device structural features such that $\sim 50 \%$ of the laser spot area is on the device, to achieve the highest responsivity from device motion to optical modulation [7]. Resonances are electrostatically excited in the devices by applying an $\mathrm{AC}$ voltage to the comb finger pads. Given the direction of the capacitive forces, in-plane resonance modes are efficiently excited.

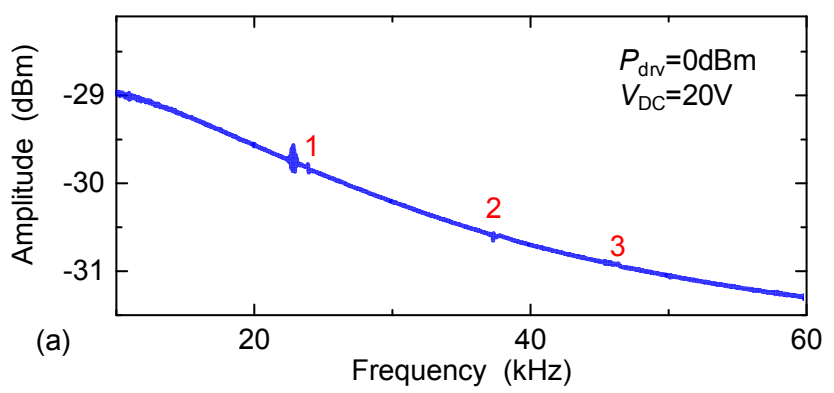

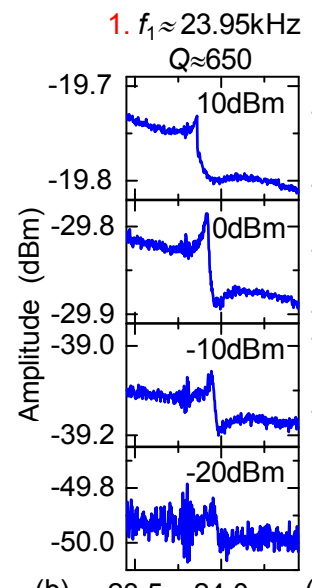

(b) $23.5 \quad 24.0$

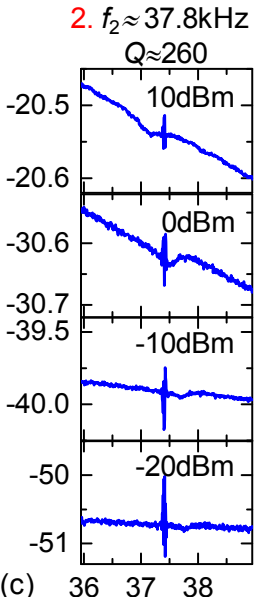

(c) $36 \quad 37 \quad 38$

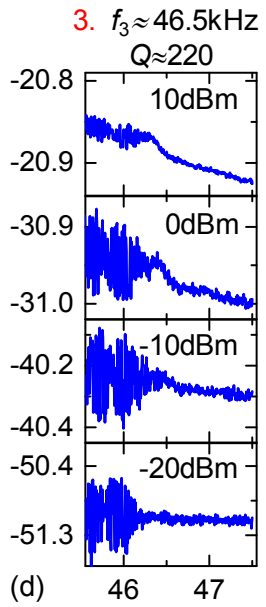

Figure 4: Measured multimode responses of the comb-drive resonator with electrical actuation and sensing. (a) The broadband response of the comb-drive resonator with 3 electrically actuated modes. (b)-(d) The 3 resonance modes, measured when $V_{D C}$ is fixed at $20 \mathrm{~V}$ and the $P_{d r v}$ is varied from $-20 \mathrm{dBm}$ to $10 \mathrm{dBm}$.

In the electrical transduction scheme, resonances of the devices are electrically detected by a simple two-port transmission measurement. The AC signal is applied to the comb fingers using a network analyzer, and the output signal from the comb-drive resonator is amplified using a low-noise voltage preamplifier with a gain of $40 \mathrm{~dB}$. In addition, a polarization DC voltage is applied to the comb-drive shuttle to investigate the electrical tunability of the resonance frequency of the comb-drive resonator.

Figure 4a shows the wide-range response of the simple twoport transmission measurement, where three resonances are readily observed. These three modes are at $f_{1} \approx 23.95 \mathrm{kHz}, f_{2} \approx 37.8 \mathrm{kHz}$, and $f_{3} \approx 46.5 \mathrm{kHz}$, with quality factors $(Q \mathrm{~s})$ of $Q_{1} \approx 650, Q_{2} \approx 260$, and $Q_{3} \approx 220$, respectively. The detection of multiple modes provides new opportunities for possible applications, such as multimode oscillators and integrated multimode resonant physical sensors. For each mode, we fix $V_{\mathrm{DC}}$ at $20 \mathrm{~V}$ and gradually increase the driving power from $-20 \mathrm{dBm}$ to $10 \mathrm{dBm}$ on the output port of the network analyzer. As shown in Fig. $4 \mathrm{~b}$ to 4 d, the amplitude of the resonance is greatly increased, and the resonance frequencies of all three modes are shifted toward lower frequencies with an increase in driving power. In addition, the first resonance mode, $f_{1}$, exhibits a clear mechanical nonlinearity at a driving power of $P_{\mathrm{drv}}=10 \mathrm{dBm}$.

Figure 5 shows the electrical frequency tuning effects by 
varying the $V_{\mathrm{DC}}$ (polarization voltage applied on the comb-drive shuttle). The DC voltage $V_{\mathrm{DC}}$ is swept from $5 \mathrm{~V}$ to $27.5 \mathrm{~V}$ for each mode and the response of the resonance is measured (see Fig. 5). For the first mode, $f_{1}$ shifts from $24.06 \mathrm{kHz}$ to $23.72 \mathrm{kHz}$ as $V_{\mathrm{DC}}$ increases from $5 \mathrm{~V}$ to $27.5 \mathrm{~V}$, exhibiting capacitive softening of the resonance frequency. In contrast, resonance frequencies of the $2^{\text {nd }}$ and $3^{\text {rd }}$ modes both upshift as $V_{\mathrm{DC}}$ increases, showing stiffening of their resonance frequencies.
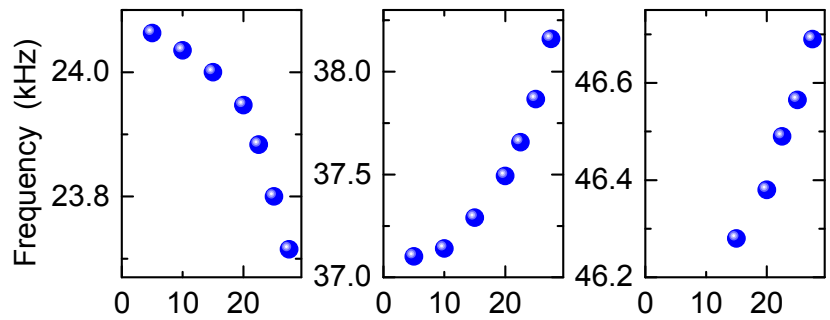

(a)

$$
V_{\mathrm{DC}}(\mathrm{V})
$$

(b) $\quad V_{D C}(\mathrm{~V})$

(c) $\quad V_{\mathrm{DC}}(\mathrm{V})$

Figure 5: $\quad$ Measured frequency tuning of 3 modes. (a)-(c) Frequency tenability as $V_{D C}$ is varied from $0 \mathrm{~V}$ to $27.5 \mathrm{~V}$.

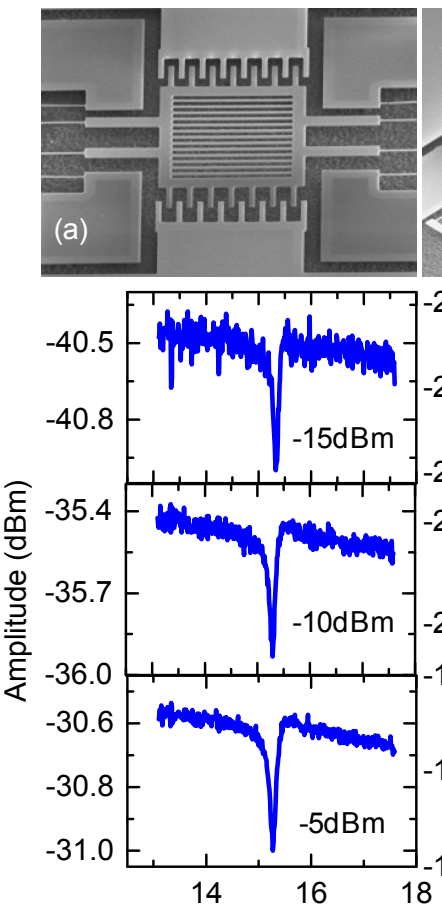

(b) Frequency $(\mathrm{kHz})$
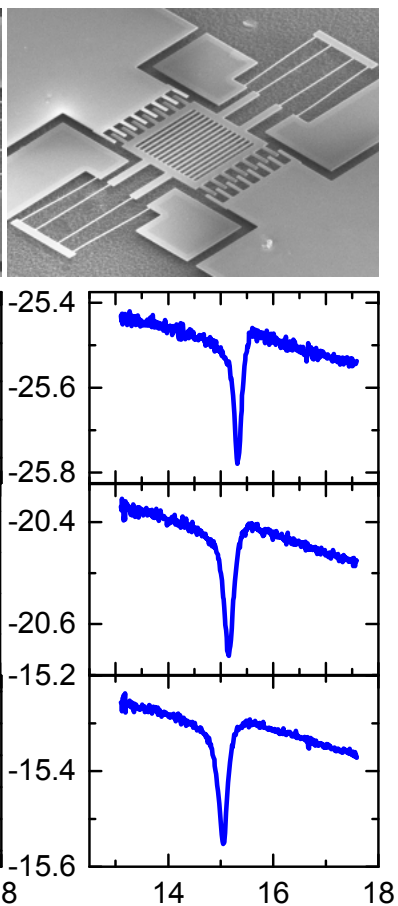

Frequency $(\mathrm{kHz})$
Figure 6: Electrically actuated comb-drive resonator with shorter folded-beam arms. (a) SEM images of the comb-drive resonator. (b) Measured resonance, with electrical readout, as the driving signal, $P_{d r v}$ is varied from $-20 \mathrm{dBm}$ to $10 \mathrm{dBm}$, at $20 \mathrm{~V} \mathrm{~V}_{D C}$.

We also investigate resonance characteristics in comb-drive resonators with folded-beam supporting arms shorter relative to the device shown in Fig. 3. We measure resonances with different driving strength from $-20 \mathrm{dBm}$ to $10 \mathrm{dBm}$ at $V_{\mathrm{DC}}=20 \mathrm{~V}$; and we find resonance frequency of $f_{1} \approx 15.10 \mathrm{MHz}$ with $Q \approx 500$.

In optical detection, we are able to measure resonances up to the $5^{\text {th }}$ mode (see Fig. 7a). Optical detection is sensitive to both inplane and out-of-plane displacements. As such, the new modes at $25.18 \mathrm{kHz}$ and $57.53 \mathrm{kHz}$ may likely be attributed to out-of-plane resonances. We test frequency tunability in the first three modes.
As with the electrical transduction scheme, we are able to optically transduce both frequency softening (Fig. $7 b$ \& $7 \mathrm{c}$ ) and frequency stiffening (Fig. 7d) behaviors as the DC voltage increases. Further, resonance amplitudes change with $V_{\mathrm{DC}}$. We find the highest resonance responses for the first three modes take place when $V_{\mathrm{DC}}$ is between $15 \mathrm{~V}$ and $20 \mathrm{~V}$. The resonance responses decrease and then increase as $V_{\mathrm{DC}}$ is swept from $22.5 \mathrm{~V}$ to $27.5 \mathrm{~V}$, for both the $2^{\text {nd }}$ and $3^{\text {rd }}$ modes. This can be explained by the 'knife-edge' effect. At low $V_{\mathrm{DC}}$, static displacement of the device is small, allowing the device to operate within the constant responsivity regime [7]. While under high DC polarization, a large static displacement changes the laser spot position relative to the device, causing the device to move outside the constant responsivity regime, hence a largely modified resonance response.

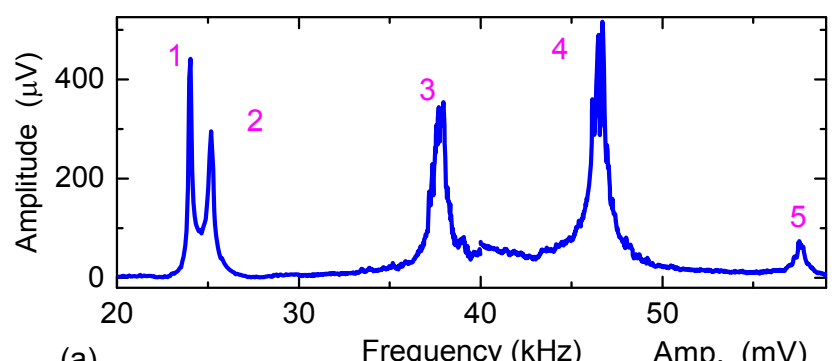

(a) Frequency $(\mathrm{kHz})$
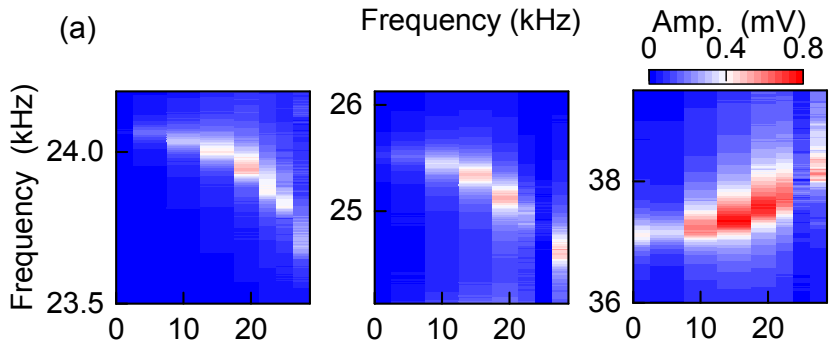

(b) $\quad V_{\mathrm{DC}}(\mathrm{V})$

(c) $V_{\mathrm{DC}}(\mathrm{V})$

(d) $\quad V_{\mathrm{DC}}(\mathrm{V})$

Figure 7: Measured resonances with optical readout. (a) The wide range spectrum. (b)-(d) Frequency tuning with $V_{D C}=0 \mathrm{~V}$ to $27.5 \mathrm{~V}$.

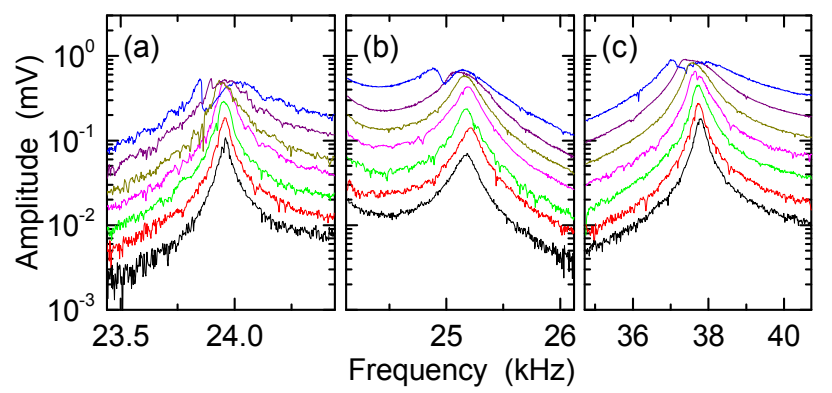

Figure 8: Electrically actuated resonances with optical readout, as $P_{d r v}$ is varied from $-20 \mathrm{dBm}$ to $10 \mathrm{dBm}$ with a step of $5 \mathrm{dBm}$. $V_{D C}=20 \mathrm{~V}$. (a)-(c) Measured data from the first 3 modes.

Next, we fix the DC polarization voltage at $V_{\mathrm{DC}}=20 \mathrm{~V}$ and gradually increase the driving force. Figure 8 shows the three optically detected resonance modes from the comb-drive resonator. Similar to the electrical transduction results, intensities of the three resonances increase as the driving strength increases. The $1^{\text {st }}$ and the $3^{\text {rd }}$ modes in the optical measurement exhibit mechanical nonlinearity when the driving strength is higher than $5 \mathrm{dBm}$, showing high power handling of these comb-drive resonators [8].

With capacitive actuation and optical readout, we observe the optical readout nonlinearity as the driving power increases: once the amplitude of resonance exceeds $600 \mu \mathrm{V}$, intensity of the signal 
decreases as the driving strength continues to increase. This is because when the displacement of the comb-drive resonator is small and within the radius of the laser spot, displacement linearly transduces into optical modulation based on the 'knife-edge' effects integrated in the interferometry; but once the displacement amplitude goes beyond the linear responsivity regime, the mechanical motion to optical signal transduction gain changes. In this latter case, the responsivity (i.e., transduction gain) from mechanical to optical transduction becomes smaller, and eventually takes on the opposite sign, thus decreasing the efficiency and gain of signal transduction. In our measured results, amplitudes of resonances show saturation near $600 \mu \mathrm{V}$ (this corresponds to a peak resonance displacement amplitude equal to the laser spot radius) which occurs at $P_{\mathrm{drv}}=0 \mathrm{dBm}$ to $5 \mathrm{dBm}$ input driving power levels. Beyond this saturation point, higher mechanical displacement decreases resonance response due to negative responsivity, leading to optical readout nonlinearity.
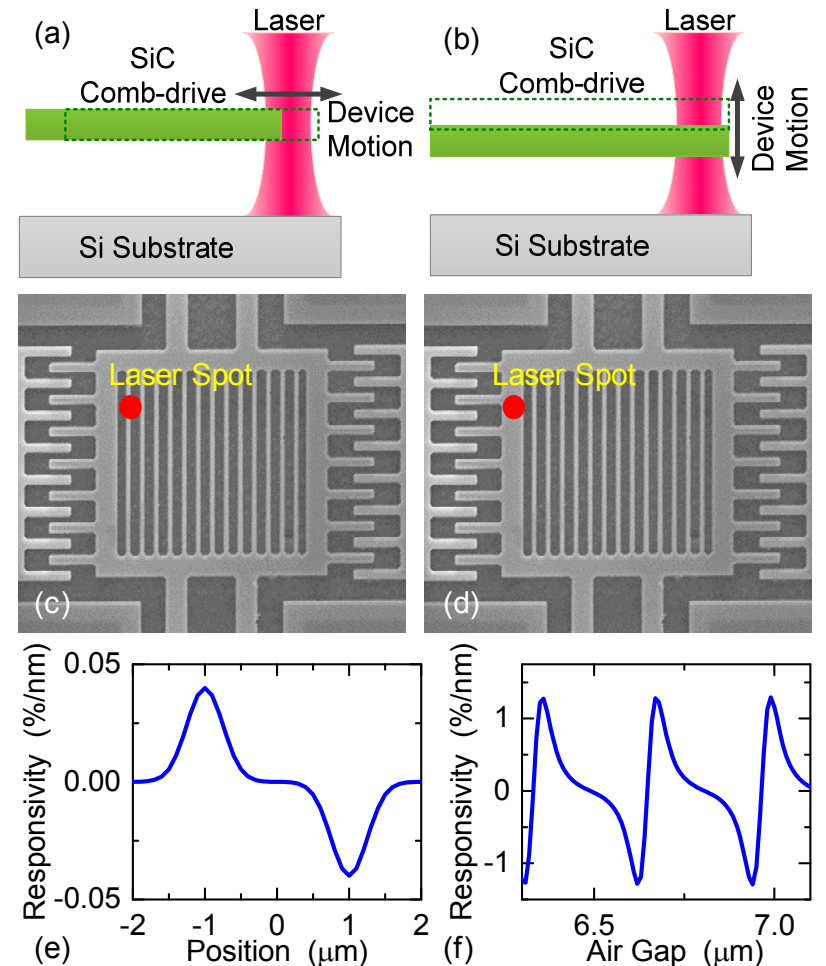

(e)

Figure 9: Illustration and analysis of transduction responsivity in the optical measurement scheme. (a)-(b) Schematic illustrations of in-plane and out-of-plane shuttle motions. (c)-(d) Laser spot positions for in-plane and out-of-plane motion detection. (e)-(f) Calculated responsivity of in-plane and out-of-plane motions.

To further investigate the nonlinearity of signal transduction in optical detection, we compute responsivity from the mechanical motion to optical signal transduction [3] (see Fig. 9) based on the actual come-drive device structures. For in-plane motions, we use the $\sim 1 \mu \mathrm{m}$ laser spot size and it is focused on the beams that comprise the inner structures of the shuttle (see Fig. 9c). For outof-plane displacement, we consider that laser spot is focused on a flat area of the shuttle (see Fig. 9d) as SiC is transparent. We find optical responsivity changes when the device oscillates with large displacements, and it takes on a different sign (e.g., negative to positive value) when device motions are larger than $\sim 1 \mu \mathrm{m}$ or $\sim 60 \mathrm{~nm}$ for in-plane or out-of-plane motions, respectively. The responsivity variation leads to saturation of measured resonances shown in Fig. 8. Our calculations can enable direct conversion from measured signal to mechanical displacement for both in-plane and out-of-plane resonant motions.

\section{CONCLUSION}

In summary, using the single crystalline $3 \mathrm{C}$-SiC-on-insulator technology, we have demonstrated $\mathrm{SiC}$ comb-drive resonators that could not be realized by conventional monolithic approaches, and investigated their resonance characteristics using a simultaneous optical and electrical displacement detection system. We have observed multimode resonances, nonlinearity in optical signal transduction, and frequency tunability. In addition, using optical detection nonlinearities arising from 'knife edge' interferometric effects, we have computed responsivity in the measurement.

\section{ACKNOWLEDGEMENT}

We thank the support from DTRA Basic Scientific Research Program (Grant No. HDTRA1-15-1-0039), NSF SNM Program (Grant No. CMMI-1246715), and Transducer Research Foundation (TRF) for the generous Student Travel Support.

\section{REFERENCES}

[1] J. Kim, A. Dutta, G. V. Naik, A. J. Giles, F. J. Bezares, C. T. Ellis, J. G. Tischler, A. M. Mahmoud, H. Caglayan, O. J. Glembocki, A. V. Kildishev, J. D. Caldwell, A. Boltasseva, and N. Engheta, "Role of Epsilon-Near-Zero Substrates in the Optical Response of Plasmonic Antennas", Optica 3, 339-346 (2016).

[2] X.-A. Fu, J. L. Dunning, C. A. Zorman, and M. Mehregany, "Polycrystalline 3C-SiC Thin Films Deposited by Dual Precursor LPCVD for MEMS Applications", Sensors \& Actuators A 119, 169-176 (2005).

[3] Z. Wang, J. Lee, and P. X.-L. Feng, "Spatial Mapping of Multimode Brownian Motions in High-Frequency Silicon Carbide Microdisk Resonators", Nature Communications 5, 5158 (2014).

[4] R. Yang, Z. Wang, J. Lee, K. Ladhane, D. J. Young, and P. X.-L. Feng, "6H-SiC Microdisk Torsional Resonators in a 'Smart-Cut' Technology", Appl. Phys. Lett. 104, 091906 (2014).

[5] J.-H. Lee, I. Bargatin, J. Park, K. M. Milaninia, L. S. Theogarajan, R. Sinclair, and R. T. Howe, "Smart-Cut Layer Transfer of Single-Crystal SiC Using Spin-on-Glass", J. Vac. Sci. \& Tech. B 30, 042001 (2012).

[6] H.-I. Kuo, C. A. Zorman, and M. Mehregany, "Fabrication and Testing of Single Crystalline 3C-SiC Devices Using a Novel SiC-on-Insulator Substrate", $12^{\text {th }}$ Int. Conf. on SolidState Sensors, Actuators \& Microsystems (Transducers'03), Boston, MA, June 8-12 (2003), pp. 742-745.

[7] J. Lee, C.-S. Li, M.-H. Li, C.-H. Chin, S.-S. Li, and P. X.-L. Feng, "Multimode Characteristics of High-Frequency CMOSMEMS Resonators", Proc. IEEE Int. Frequency Control Symposium (IFCS'14), Taipei, Taiwan, May 19-22 (2014), pp. 478-480.

[8] X. Liu, L. P. B. Katehi, W. J. Chappell, and D. Peroulis, "Power Handling of Electrostatic MEMS Evanescent-Mode (EVA) Tunable Bandpass Filters", IEEE Trans. Microwave Theory \& Techniques, 60, 270-283 (2012).

\section{CONTACT}

*P. X.-L. Feng, tel: +1-216-368-5508; philip.feng@,case.edu ${ }^{2}$ Present: Air Force Research Laboratory, Dayton, OH 45433.

${ }^{3}$ Present: LakeShore Cryotronics, Inc., Westerville, OH 43082. 